\title{
Engaging Higher Order Thinking Skills with a Personalized Physics Tutoring System
}

\author{
Matthew Bojey ${ }^{1}$, Bowen $\mathrm{Hui}^{1}$, and Robert Campbell ${ }^{2}$ \\ 1 Department of Computer Science, University of British Columbia \\ 2 Faculty of Education, University of British Columbia
}

\begin{abstract}
Recent research shows a lack of student interest and declined enrollment in physics. Our system offers four levels of difficulty with activities that enable students to exercise a range of lower and higher order cognitive skills. Moreover, we adopt existing methods in probabilistic user modeling to provide personalized help. Our work models both domain concepts as well as user attitudes.
\end{abstract}

Keywords: Probabilistic user modeling, dynamic Bayesian networks, higher order cognitive skills, Bloom's taxonomy, physics education

\section{Introduction}

Research shows a declined enrollment in university physics programs and that physics as taught in schools do not seriously take student interests into account [4]. As a way to overcome this challenge, attempts to integrate physics material in an interactive and individualized manner have shown to increase student interest and performance (e.g., $[3,5])$. However, students' interest in physics is closely related to their self-esteem and sense of academic achievement [4]. Fostering student interest requires teachers to pay close attention to students and guide them. Unfortunately, large-sized classes make it logistically infeasible to realize this. We propose an intelligent tutoring system (ITS) that provides individualized feedback and aims to increase student interest in physics by providing a variety of activities that exercise different levels of thinking skills.

Originally devised as handbooks to systematize learning objectives and assessment, Bloom's taxonomy has become a foundational structure in Education [1]. The taxonomy reprsents the process of mastering a subject through several levels of cognitive activities starting wtih remembering at the lowest level and creating at the highest.Our objective in this work is to adopt Bloom's taxonomy to create interactive activities that enable students with varying expertise to apply a range of cognitive skills.

\section{System Overview}

Our system is called Kirchhoff's Rules Intelligent Tutor (KRIT) as it focuses on helping students with the application of Kirchhoff's rules. The complexity of a 
circuit is determined by the layout parameters (number of batteries, resistors, and junctions). The objective of these exercises is to apply Kirchhoff's rules and algebraically solve for one of three variables (voltage, resistance, current). KRIT has four activity types displayed as separate levels to the student, multiple choice questions, coached exercises, guided exercises and a create and share activity.

Our student model represents how much help a student currently needs as well as thier current level of understanding. These factors are crucial in developing a personalized ITS because different types of students prefer different levels of assistance regardless of their level of understanding. These preferences may also vary as a function of the exercises' difficulty. Hence, a personalized tutor must provide support suited for the individual's needs.

Since this type of information is unknown to KRIT, it must be inferred indirectly. Due to the inherent uncertainty of the inference problem, we adopt a probabilistic approach by using a dynamic Bayesian network (DBN) [2]. Our DBN reflects how the student's domain knowledge ( $K$ for short) influences her performance on applying algebra and physics concepts. We use observable events to estimate the student's understanding of these concepts thus, the DBN incorporates all the events observed from one response at each stime step.

To model student characteristics, our DBN includes the current need for help and receptiveness to help ( $N$ and $R$ for short respectively). We estimate these variables using passively collected behavioral observations such as pausing, undoing what was typed, and making use of hints and system explanations. Additionally, note that $K$ influences $N$ to model advanced students are less likely to need help. In turn, $N$ influences $R$ to model the correlation between neediness and receptiveness to automated help. Together, $K$ and $N$ define the student's current state which is used to inform KRIT in its decision making.

\section{Conclusions and Future Work}

Pilot studies have shown promising results and we are currently in the process of performing a large scale usablity test. We also have plans to develop the system for mobile platforms and perform a longitudinal study.

\section{References}

1. B.S. Bloom. Taxonomy of Educational Objectives, Handbook I: The Cognitive Domain. David McKay Company, Inc., New York, 1956.

2. T. Dean and K. Kanazawa. A model for reasoning about persistence and causation. 5(3):142-150, 1989.

3. A .Gertner and K. VanLehn. Andes: A coached problem solving environment for physics. pages 133-142, 2000.

4. P. Häussler and L. Hoffmann. A curricular frame for physics education: Development, comparison with students interests, and impact on students achievement and self-concept. Science Education, 84:689-705, 2000.

5. L.S. Myneni and N.H. Narayana. An Intelligent Tutoring and Interactive Simulation Envioronment for Physics Learning. pages 250-255, 2012. 